\title{
Organic emitters for solid state lighting
}

\author{
G. M. Farinola* and R. Ragni
}

\author{
* Correspondence: \\ gianlucamaria.farinola@uniba.it \\ Dipartimento di Chimica, Università \\ degli Studi di Bari "Aldo Moro", via \\ Orabona 4, I-70126 Bari, Italy
}

\begin{abstract}
White organic light emitting diodes (WOLEDs) have gained considerable attention of academic and industrial research communities as promising alternative to incandescent lamps, fluorescent tubes and inorganic LEDs for low energy consumption lighting applications. WOLEDs are expected to become one of the next generation lighting sources because of their high color tunability and color quality, which can be easily achieved by proper chemical design of organic electroluminescent materials. Contrary to their inorganic counterparts, WOLEDs also bear the distinctive feature of being available as flexible and large area devices. Over the last decades, a lot of efforts have been spent on defining suitable strategies to highly efficient WOLEDs based on the use of properly tailored organic emitters. Here we survey the main chemical approaches to white electroluminescence from organic light emitting materials, highlighting strong and weak points of each strategy. Current research on new hybrid white light emitting devices based on the combination of inorganic LEDs and organic down color converters is also reviewed by some representative examples.

Keywords: White OLEDs; White electroluminescence; Organic fluorescent molecules; Organic conjugated polymers; Phosphorescent organometallic complexes; Organic down converters; LUCO dyes
\end{abstract}

\section{Introduction}

Solid state lighting (SSL) sources based on light emitting diodes represent the new generation of highly efficient illumination systems with significant impact on energy saving.

For organic and inorganic light emitting diodes (OLEDs and LEDs) the luminous power efficiency, defined as the output light power from a device per electrical power input, has respectively reached and overcome the value of $100 \mathrm{~lm} \mathrm{~W}^{-1}[1,2]$, which is comparable to that of fluorescent lamps $\left(65-100 \mathrm{~lm} \mathrm{~W}^{-1}\right)$ and much higher than that of incandescent light bulbs $\left(15 \mathrm{~lm} \mathrm{~W}^{-1}\right)[3,4]$.

OLEDs generate light relying on electroluminescence from organic or organometallic molecules. They generally have a multilayer architecture in which a thin film of one or more light emitting materials is sandwiched between two thin electrodes, one of which at least must be transparent to light. Further charge transporting (HTL, ETL) or blocking (HBL) layers are deposited between the electrodes and the emissive zone, in order to improve device performance.

Applying a voltage between the electrodes, electrons and holes are injected from the cathode and the anode respectively and, under the influence of the external electric field, they easily across the charge transporting layers and recombine in the emitting 
material, forming electron-hole pairs (excitons) responsible for light generation by radiative decay.

Contrary to the inorganic counterpart, WOLED technology allows fabrication of large area, flexible and variously shaped lighting sources, due to the easy film processing of organic electroluminescent materials on substrates, such as plastics, with various techniques as vacuum thermal evaporation, spin coating or casting from solution. Vacuum thermal evaporation represents the layer deposition method currently used in close to $100 \% \mathrm{com}$ mercially available OLED displays and lighting products: it allows higher layer complexity and stability with respect to the layer processing techniques from solution which, on the other side, have gained the attention of materials scientists because of their lower cost.

Other appealing features of WOLEDs are the possibility to achieve high brightness at low operating voltage, as well as to finely tune light color quality by tailored molecular design of organic light emitting materials [5-7].

Although LED-based lighting technology is more mature than the organic alternative, which must still fulfill requirements of performance and cost for being competitive in general illumination market, WOLED efficiencies have been predicted to rapidly increase in the next years, reaching values of $200 \mathrm{~lm} \mathrm{~W}^{-1}$ similar to those expected for inorganic LEDs [8].

The possibility to lead WOLEDs to the level of broadly diffused lighting sources is also related to the development of new organic emitting materials with improved stability and efficiency: therefore, research efforts in the design, synthesis and combination of efficient organic and organometallic emitters represent the heart of the progress of WOLED technology.

Moreover, in the next two years, a new hybrid technology based on the deposition of organic luminescent color converters (LUCO) on top of an inorganic LED is gaining momentum: this approach allows to combine high efficiencies typical of inorganic LEDs with the high color quality and cheap processing techniques of organic emitters. Therefore, it is expected to strongly influence the future of commercial lighting sources.

\section{Review}

\section{Main strategies to white electroluminescence from organic emitters}

The basic principle for generating white electroluminescence lies in the combination of moieties that simultaneously emit light of two complementary (orange and blue) or three fundamental (red, green and blue) colors. However, combination of emitters of different colors requires proper control of energy transfer processes that funnel excitons from the highest energy emitters (blue) to the lowest ones (orange/red). In order to have simultaneous light production from all emitters, a fine control of their relative amount and interaction is necessary on both the molecular and the device scale.

White electroluminescence from organic materials can be pursued by various approaches mainly classified into two categories $[2,9]$. The first one involves the combination of two or more emitters of different colors that can be (1) confined in different stacked layers or (2) blended in a single emitting layer of WOLED. Further approaches rely on a single material whose emission, covering a significant part of the visible spectrum, results (3) from the excited states of individual molecules and of their aggregates (excimers and electromers) in the solid state, or (4) from different color 
emitting moieties covalently bound in a single macromolecular structure. Finally, white light can be also generated by (5) a blue inorganic LED bearing an external down conversion layer of organic dye.

Aside from the approach used, organic materials emit light by fluorescence radiative decay, typical of $\pi$-conjugated polymers and organic small molecules $[10,11]$, or by phosphorescence occurring in organometallic complexes of Ir, Pt, Ru, Os and some lanthanides $[12,13]$. These different radiative decay pathways affect device performances. In particular, while fluorescent materials usually convert into light only singlet excitons (i.e. $25 \%$ of all excitons generated by electron-hole recombination), phosphorescent organometallic complexes generate light from both singlet $(25 \%)$ and triplet $(75 \%)$ excited states, leading to higher device efficiencies [14]. However, very recent studies have demonstrated the possibility to achieve high external quantum efficiencies also for WOLEDs made with some organic fluorophores having thermally activated delayed fluorescence (TADF), due to the reverse intersystem crossing (RISC) of triplet excited states into singlet excitons [15].

Some representative examples highlighting advantages and issues of the various approaches to white organic electroluminescence will be discussed in the next paragraphs and, although their selection has not been made on the basis of the best performances affordable by each approach, the whole data set of devices here reported is summarized in Table 1.

\section{Approach (1): WOLEDs based on emitters stacked in a multilayer structure}

White electroluminescence can be achieved by stacking multiple layers of fluorophores or phosphors which emit light of complementary (blue and orange) or fundamental (red, green, blue) colors. These emitters are generally dispersed as "dopants" in charge transporting host materials.

The main advantages of multilayer WOLEDs are that the thickness or dopant concentration of each layer can be individually optimized to improve the white color purity. Nevertheless, the multiplicity of layers adds complexity to device manufacture and the high overall thickness of the emitting zone leads to operating voltages superior to those required for WOLEDs based on single emitting units. Other drawbacks are that the emission color of device is voltage dependent and, in the case of solution processed stacked WOLEDs, the successive deposition of each layer is detrimental to the surface morphology of the preformed emissive layer which can be dissolved and mixed to the upper one. A possible way to overcome this issue is to use materials with different solubility and to stack them by orthogonal solvents $[16,17]$.

For instance, a white organic light emitting device can be obtained stacking the blue emitting copolymer LEP deposited by the hydrophobic mesitylene solvent on the top of an hydrophilic layer of the orange emitting iridium complex iTMC (Fig. 1a) [18].

White light is also reported for devices based on three stacked emissive layers of the red $(\mathrm{PPQ})_{2} \operatorname{Ir}(\mathrm{acac})$, green $\operatorname{Ir}(\mathrm{ppy})_{3}$ and blue FIrpic phosphorescent iridium complexes doped in the same host material (Fig. 1b) [19]. In this case, external quantum efficiency up to $20 \%$ and desired output color balance can be achieved by controlling both the thickness and the relative position of the emitting layers.

Very high performances have been recently recorded also for WOLEDs made with stacked layers of red, green and blue emitting fluorophores showing thermally activated 
Table 1 Data set of devices

\begin{tabular}{|c|c|c|c|c|c|c|c|}
\hline Ref. & Figure & $\eta_{\text {ext }}(\%)$ & Power efficiency $\left(\mathrm{ImW}^{-1}\right)$ & Luminous efficiency $\left(\mathrm{cdA}^{-1}\right)$ & CRI & $\mathrm{CCT}(\mathrm{K})$ & $\mathrm{ClE}$ \\
\hline 18 & $1 \mathrm{a}$ & - & - & $0.46,0.90$ & - & - & $(0.324,0.337)$ \\
\hline 19 & $1 b$ & 20.1 & 41.3 & - & 85 & - & $(0.38,0.39)$ \\
\hline 20 & 2 & 17 & 34.1 & 45.6 & - & - & $(0.33,0.41)$ \\
\hline \multirow[t]{2}{*}{21} & 3 & 21 & - & - & - & - & $(0.31,0.33)$ \\
\hline & & 22.9 & - & - & - & - & $(0.39,0.43)$ \\
\hline 22 & 4 & 10.5 & 3.31 & - & 13 & 5899 & $(0.33,0.31)$ \\
\hline 23 & 5 & 3.1 & - & 3.7 & - & - & $(0.32,0.33)$ \\
\hline 24 & 6 & - & 8.9 & 9.8 & 91 & 4632 & $(0.36,0.37)$ \\
\hline 25 & 7 & 3.2 & - & 12.5 & 86 & - & $(0.34,0.34)$ \\
\hline 26 & 8 & 19.1 & 20.3 & 42.9 & 55 & 4146 & $(0.395,0.452)$ \\
\hline 27 & $9 a$ & 0.35 & - & - & - & - & $(0.31,0.42)$ \\
\hline 28 & $9 b$ & 15.9 & 12.6 & 37.8 & 69 & - & $(0.46,0.47)$ \\
\hline 29 & 10 & 3 & 5.14 & 5.4 & 86 & - & $(0.33,0.33)$ \\
\hline 30 & $11 a$ & 3.8 & 5.75 & 8.99 & - & - & $(0.35,0.34)$ \\
\hline 31 & $11 b$ & - & - & 0.71 & - & - & $(0.31,0.39)$ \\
\hline 32 & $12 \mathrm{a}$ & 5.4 & - & 10.7 & - & - & $(0.37,0.30)$ \\
\hline 33 & $12 b$ & 3.7 & 7.2 & 8.2 & 82 & - & $(0.35,0.38)$ \\
\hline 34 & 13 & 6.28 & 7.73 & 16.62 & - & - & $(0.33,0.36)$ \\
\hline 35 & 14 & - & 2.4 & 6.7 & 88 & - & $(0.33,0.35)$ \\
\hline 37 & 15 & - & 13.6 & - & - & 5137 & $(0.31,0.31)$ \\
\hline 38 & 16 & 90 & - & - & 80 & 5980 & $(0.32,0.33)$ \\
\hline 39 & 17 & 45.4 & 123.8 & - & - & - & $(0.32,0.33)$ \\
\hline
\end{tabular}

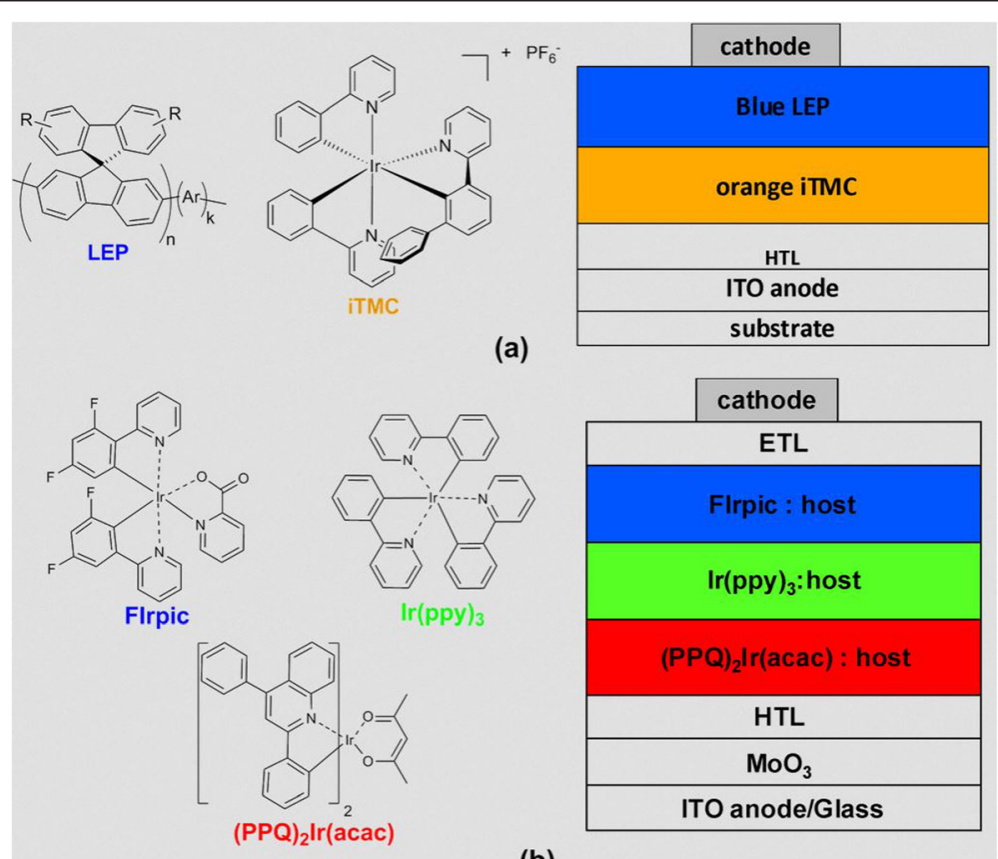

(b)

Fig. 1 WOLEDs made with a blue and orange, b blue, green and red stacked emitting layers 
delayed fluorescence (TADF) [15]. In TADF, triplet excitons of a fluorescent dye can be up-converted to singlet excitons by efficient reverse intersystem crossing (RISC), this preventing non radiative decay from the triplet states. In particular in 2014, Nishide et al. designed a multilayer WOLED device in which three stacked emitting layers are used: (1) a layer of the TADF green emitting molecule 4CzPN doped in mCBP [3,3-di(9H-carbazol-9-yl)biphenyl] host, (2) a layer containing both 4CzPN and the red TADF dopant 4CzTPN-Ph in mCBP and (3) a layer of the TADF blue dye 3CzTRZ in PPT [2,8-bis(diphenylphosphoryl)dibenzothiophene] host (Fig. 2) [20]. The resulting device has CIE coordinates of $(0.30,0.38)$ and external quantum efficiency over $17 \%$.

In 2015, Cho et al. have also reported highly efficient WOLEDs based on the blue DCzIPN TADF dye acting both as a blue emitter and a host for the yellow iridium phosphor PO-01 (Fig. 3) [21]. In particular, the device architecture includes three stacked layers of DCzIPN, the central one embedded with the yellow emitting PO-01 complex (Fig. 3). Interestingly, the authors report that it is possible to manage electroluminescence from warm to cool white color by properly changing the thickness of the central yellow emitting layer. High external quantum efficiencies (21.0 \% and $22.9 \%)$ have been observed for the cool and the warm white light emitting devices, respectively showing CIE coordinates $(0.31,0.33)$ and $(0.39,0.43)$.

Another approach to efficient and stable white electroluminescence consists in stacking two or more single devices which emit light of different colors. Despite their complex manufacturing process, these architectures, also known as tandem WOLEDs, have the advantage to achieve high brightness at low current density.

For instance, CIE coordinates $(0.33,0.31)$ and luminance of $4450 \mathrm{~cd} / \mathrm{m}^{2}$ are observed just at $17 \mathrm{~V}$ operating voltage for the tandem WOLED reported in Fig. 4. This device has a multilayer architecture in which an interfaced anode-cathode layer (ACL) is not

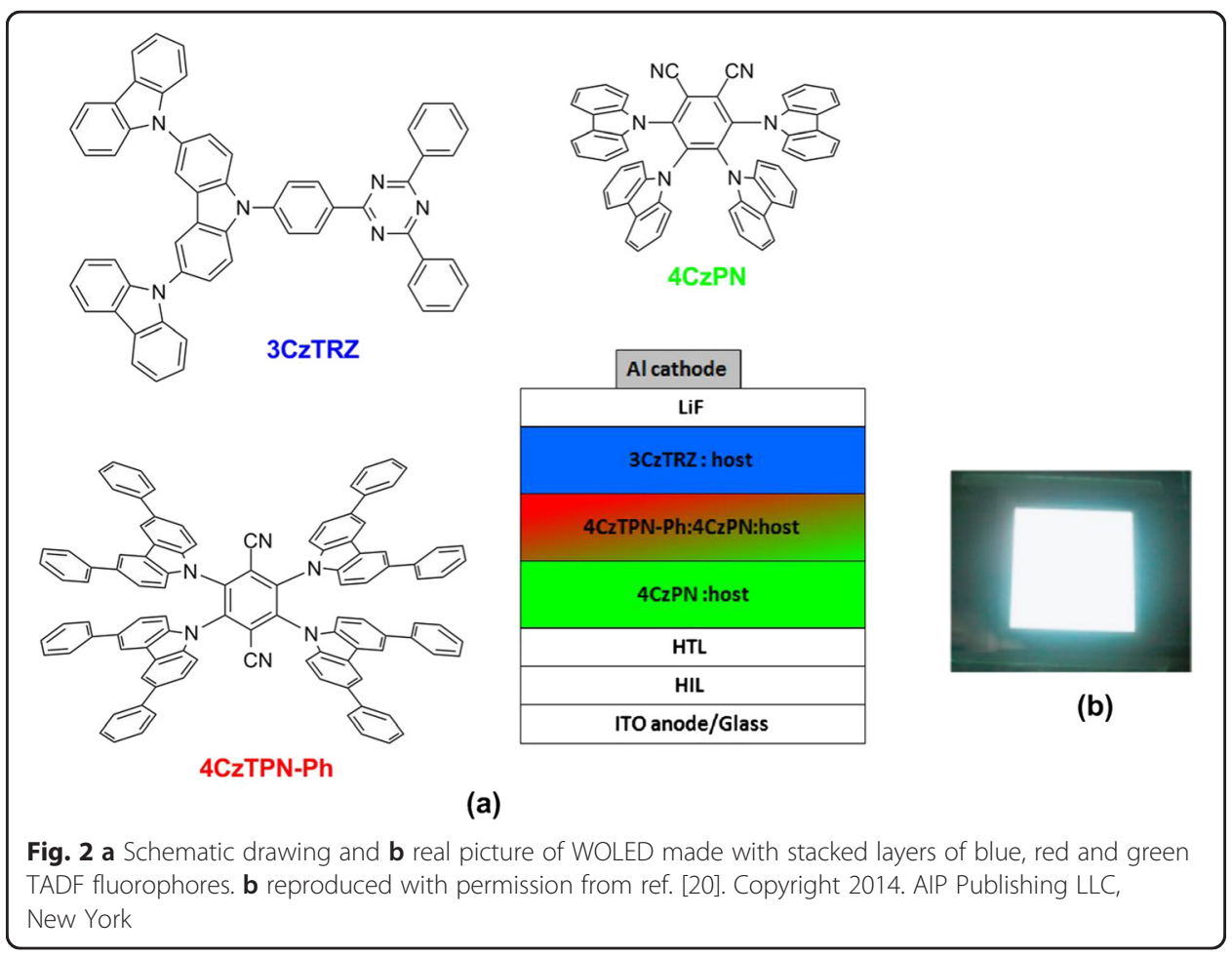


<smiles>N#Cc1cc(-n2c3ccccc3c3ccccc32)c(-n2c3ccccc3c3ccccc32)cc1C(N)=NC1CCCCC1</smiles>

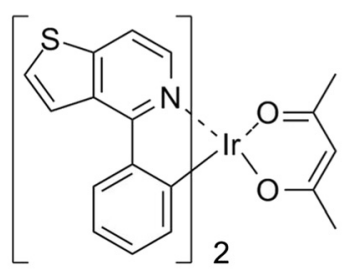

PO-01

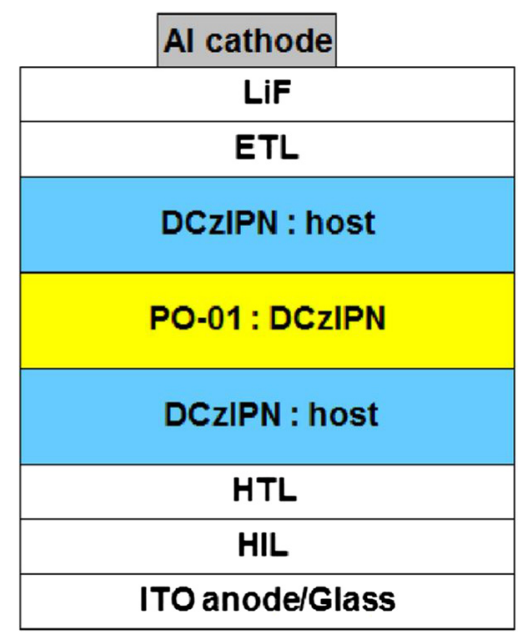

Fig. 3 Schematic drawing of WOLED made with stacked layers of blue and yellow TADF fluorophores

connected to the power source and acts as the electron injector for the bottom device relying on the blue emission of the FIrpic iridium complex and as the hole injector for the top OLED made with the red [Btp $\left.\mathrm{P}_{2} \operatorname{Ir}(\mathrm{acac})\right]$ emitter [22].

\section{Approach (2): WOLEDs bearing multiple emitters in a single layer}

White electroluminescence from a single emitting layer device can be achieved by two methods: blending polymers with two complementary or three fundamental emission colors, or doping a wide energy gap host material with small percentages of lower energy phosphorescent emitters. Both methods are suitable for low cost large area WOLED fabrication since combining multiple color emission in a single layer allows easy processing from solution or evaporation techniques. However, a careful control of morphology and composition of the emitting layer is fundamental to manage energy transfer processes responsible for white light output. The major drawback of this approach versus that based on stacked emitting layers is that it is more difficult to compensate the differential aging of emitters blended with a precise molar ratio in a single emitting layer. In particular, in WOLEDs made of binary or ternary blends, the control

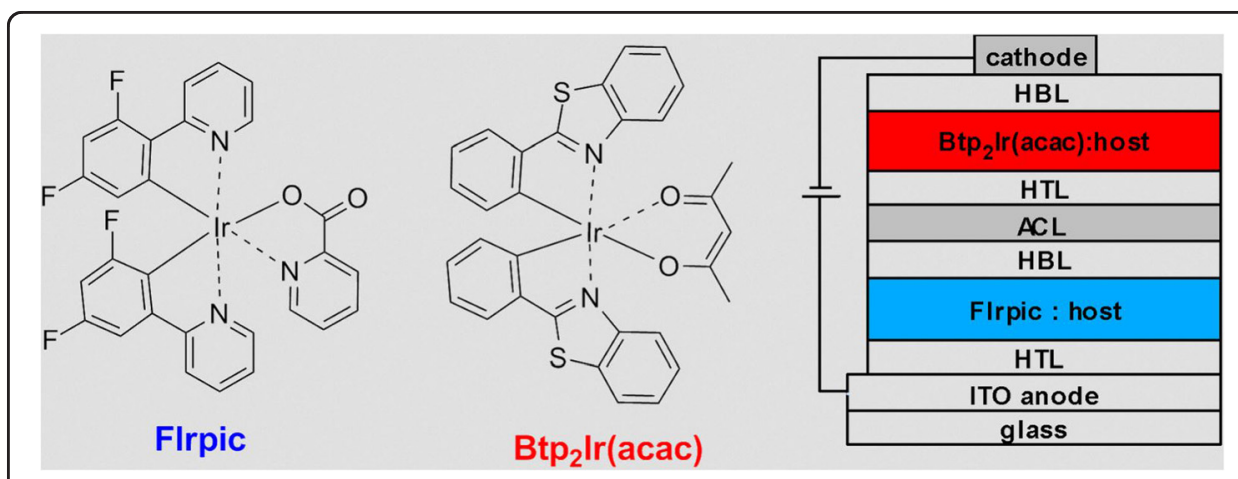

Fig. 4 White electroluminescence from stacked red and blue OLEDs 
of emitters ratio is less critical than in doped systems but, their efficiency and brightness are lower since singlet emitters are generally used.

A representative example of the first method is a WOLED made of a binary blend of blue and orange-red emitting polymers (PFO and MEH-PPV reported in Fig. 5) [23].

In particular, to get white light by simultaneous emission of both polymers, the energy transfer from PFO to MEH-PPV must not be complete. This occurs for blends with MEH-PPV:PFO composition in 5-28 wt-\% range which have morphology characterized by phase segregated nanocrystalline PFO domains and amorphous MEH-PPV domains. Indeed, for higher MEH-PPV percentages, the blend is amorphous and complete energy transfer from PFO to MEH-PPV leads to the sole orange-red electroluminescence.

A single layer WOLED with excellent color rendering index and stability has been also recently obtained by blending in proper ratio blue (PPF-SO), green (PPF-SO-BT) and red (PPF-SO-DHTBT) light emitting copolymers bearing dibenzothiophene-S, Sdioxide moieties (Fig. 6) [24]. In this case white light stability has been attributed to the fact that the three copolymers have very similar chemical structures and do not show tendency to crystallization and phase separation.

In WOLEDs based on a single emitting layer of a host-dopant guest system, the host is responsible for charge transport and, in some cases, for blue emission while phosphorescent dopants emit light by direct charge trapping or energy transfer from the host. For instance, an efficient WOLED has been fabricated by doping the blue emitting BlueJ polymer with the green $\left[\operatorname{Ir}(\mathrm{PBPP})_{3}\right]$ and the red $\left[\operatorname{Ir}(\mathrm{PIQ})_{3}\right]$ iridium complexes (Fig. 7) [25]. Also in this case the role of the emitting layer morphology is crucial to achieve white electroluminescence: indeed, doping iridium complexes in the sole BlueJ does not lead to white light since the poor miscibility of these materials completely prevents energy transfer from the blue host to the guests. This drawback is overcome using a further non emitting polymer, the polyvinylcarbazole (PVK), which improves either hole mobility and miscibility of the other components, thus generating a microphase-separated system that favors a cascade partial energy transfer from the PVK/BlueJ matrix to the two iridium phosphors.

Moreover, white-yellow electroluminescence suitable for lighting applications has been achieved by doping PVK with proper percentages of the sky-blue FIrpic, the yellow Ir(DPA-Flpy) $)_{3}$ complex and the electron transporting OXD-7 material (Fig. 8) [26].

\section{Approach (3): WOLEDs based on single molecular emitters}

The development of devices composed of a single white emitting molecular component is strongly desirable to reduce costs and simplify manufacture, as well as to avoid the accurate control of molar ratio of multiple emitters used in the approach (2).

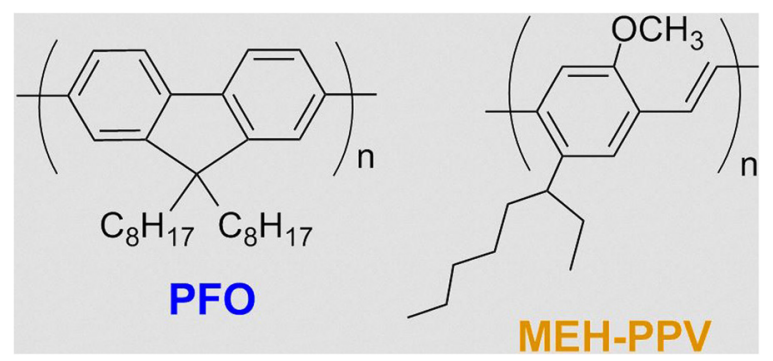

Fig. 5 Chemical structures of the blue PFO and orange MEH-PPV emitting polymers 


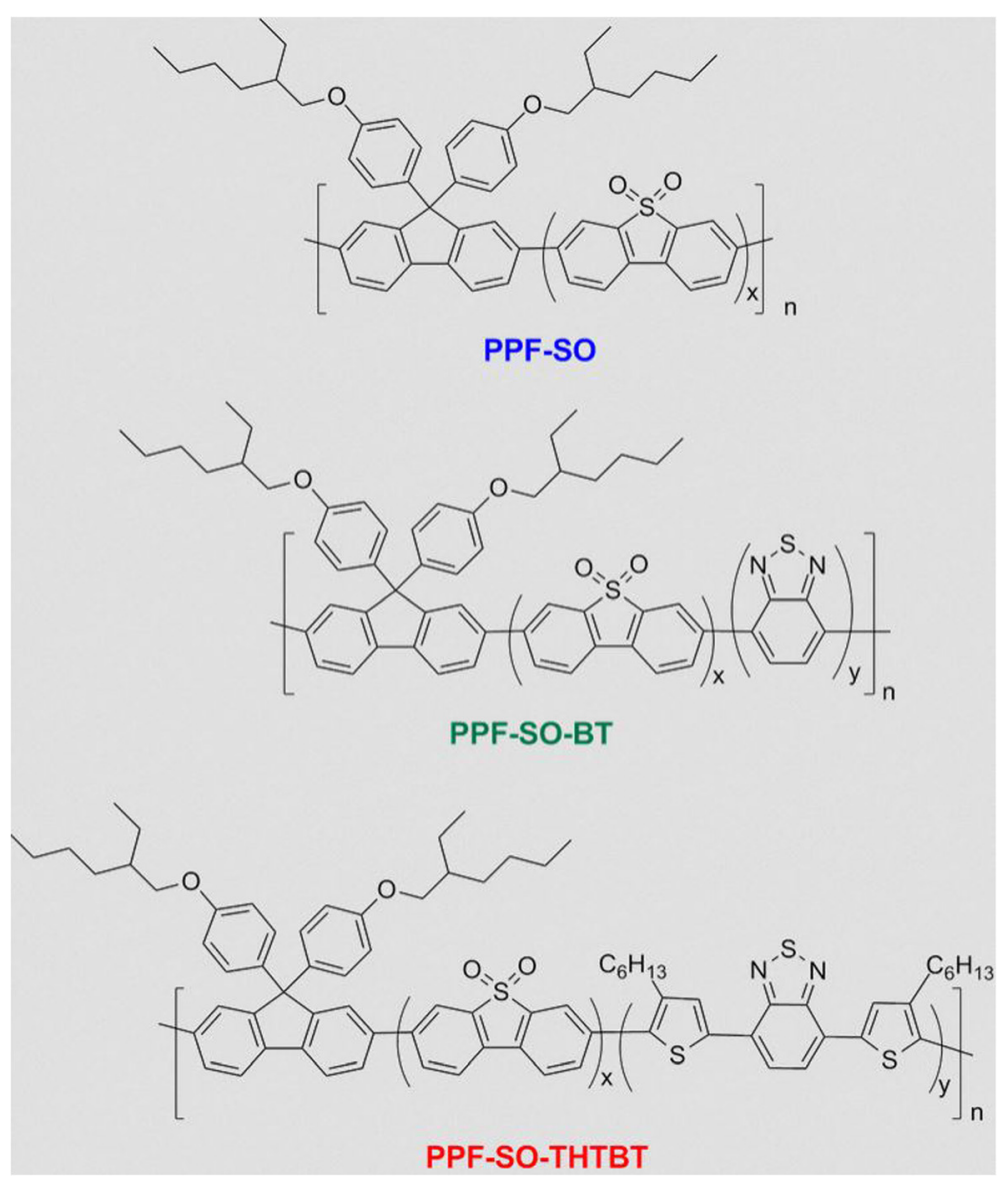

Fig. 6 White electroluminescence from WOLED made of a ternary blend of blue, green and red emitting copolymers

A possible way to attain this target involves the use of molecules that simultaneously emit blue light from their individual excited state and red-orange light from their excited aggregates (excimers or electromers) generated in the solid state. In this approach, despite the less complex composition of the emitting layer, since only one species is responsible for both blue and orange-red light, possible drawbacks are the low efficiency and the difficult molecular design of the emitter.

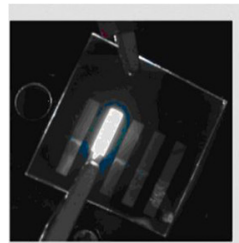

(a)

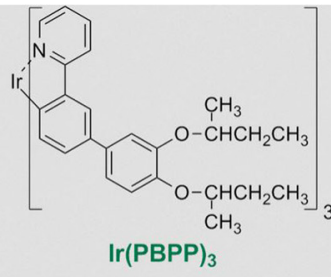

$\operatorname{rr}(\mathrm{PBPP})_{3}$

Fig. 7 a Photo of WOLED made doping green $\operatorname{Ir}(\mathrm{PBPP})_{3}$ and $\operatorname{red} \operatorname{Ir}(\mathrm{PIQ})_{3}$ phosphorescent emitters in a single host layer composed of blue emitting BlueJ and hole transporting PVK polymers. b Chemical structures of materials used in the emitting layer. a reproduced with permission from ref. [25]. Copyright 2006, WILEY-VCH Verlag GmbH \& Co. KGaA, Weinheim 


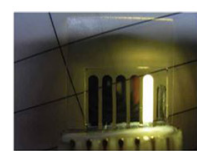

(a)

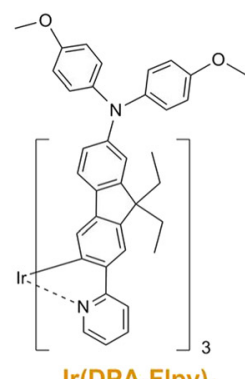

$\operatorname{Ir}(\text { DPA-FIpy })_{3}$

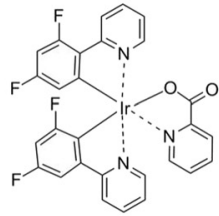

FIrpic

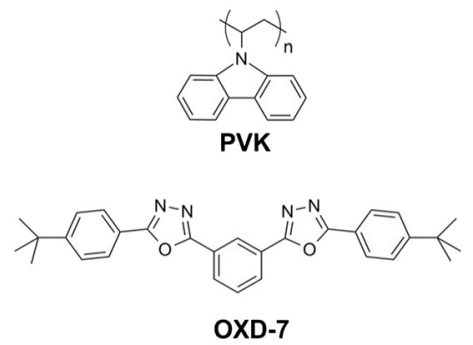

(b)

Fig. 8 a Photo of WOLED based on a single PVKNOXD-7 host layer doped with blue Flrpic and yellow Ir(DPA-Flpy) ${ }_{3}$ iridium phosphors. $\mathbf{b}$ Chemical structures of materials used in the emitting layer. a reproduced with permission from ref. [26]. Copyright 2009, WILEY-VCH Verlag GmbH \& Co. KGaA, Weinheim

For instance, a terthiophene based emitting compound (Fig. 9a) bearing methyl and mesityl substituents has been found to emit white light when used as the sole active material in a very simple device architecture (ITO/HTL/active layer/cathode) [27]. White electroluminescence results from the superimposition of the blue-green terthiophene monomer emission and the red emission of an excimer having a well-defined cross-like conformation which is due to the sterical hindrance existing between the methyl and the mesityl groups of two neighbouring monomers that form the excimer. In particular, the presence of both methyl and mesityl groups in proper positions of the monomer is fundamental to get white light because, in their absence, the cross-like excimer is not generated and no red emission can be observed in the aggregate state.

Warm white electroluminescence has been also achieved by a blue phosphorescent platinum complex (FPt in Fig. 9b) that, due to its square planar structure, generates orange emitting excimers when incorporated in a host non emitting layer [28].

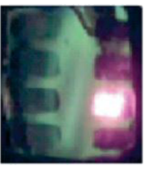<smiles></smiles>

(a)
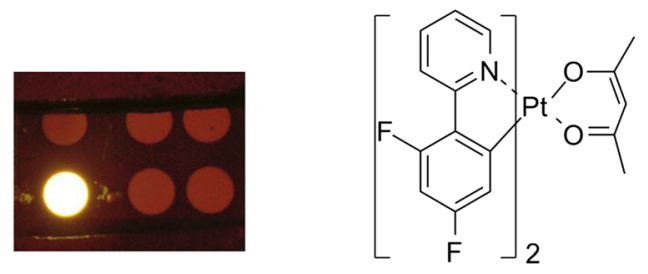

FPt

(b)

Fig. 9 White WOLED based on a terthiophene molecule and $\mathbf{b}$ a platinum phosphorescent complex. $\mathbf{a}$ and $\mathbf{b}$ adapted with permission from ref. [27] and ref. [28]. Copyright 2005 and 2007, WILEY-VCH Verlag $\mathrm{GmbH} \&$ Co. KGaA, Weinheim 
Besides the use of molecules that simultaneously emit light from their individual excited state and their excited aggregates, white electroluminescence can be also achieved by molecules showing completely different emission color from their neutral and protonated or deprotonated forms. In particular, a molecular dyad with a blue emitting neutral form composed of an electron donor (D) and a weakly electron acceptor (A) unit can be converted, by controlled acid stimulation, into its orange emitting protonated form [29]. This significant change of color emission is due to a proton induced enhancement of the electron accepting ability of the A unit that strengthens intramolecular charge transfer from $\mathrm{D}$ to $\mathrm{A}$ inside the molecular dyad. White light is the result of simultaneous blue and orange emission from the neutral and the protonated forms by proper control of doped acid amount which regulates conversion from the blue to the orange dye structures. WOLEDs fabricated in 2013 by Liu et al. doping the emitting layer of the neutral blue dye in Fig. 10 with a small amount of camphorsulfonic acid (CSA) showed external quantum efficiency of $3 \%$, which is a significant result if we consider the simplicity of both material design and device fabrication [29].

\section{Approach (4): WOLEDs based on polymers bearing multiple color emitting moieties}

White electroluminescent copolymers bearing singlet or triplet emitting units of different colors are of particular interest since they allow to avoid interfacial mixing observed for emitters stacked in multiple layers approach (1) and intrinsic phase separation typical of blends or host-guest systems used in the approach (2). However, this class of materials is synthetically challenging since a very careful control of relative amount and connection of the single emitting units is fundamental to manage energy transfer processes occurring in the polymer. Moreover, efficiencies reported so far for devices based on this approach are generally lower than those observed for multilayer stacked WOLEDs.

The most used strategy for the synthesis of white emitting copolymers relies on the covalent linking, in the main chain or as side groups, of light emitting moieties with complementary or fundamental colors. Some representative structures of white electroluminescent copolymers bearing blue and orange or blue, red and green fluorescent units combined in the main chain are reported in Figs. 11a and b, respectively. In both cases, the molar ratio of monomers plays a key role in control of polymer emission [30, 31].

High efficiencies have been found for white emitting copolymers bearing phosphorescent complexes in the main chain or as pendant groups [32, 33]. For instance low

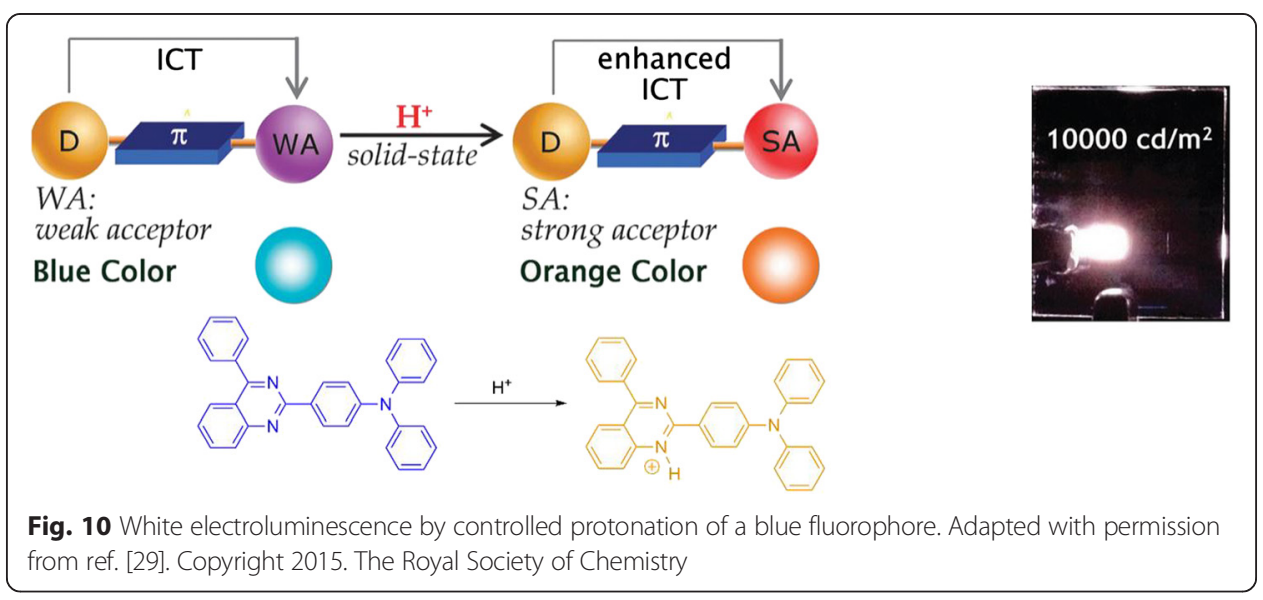




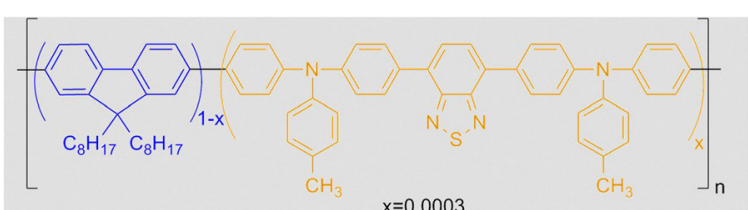

(a)

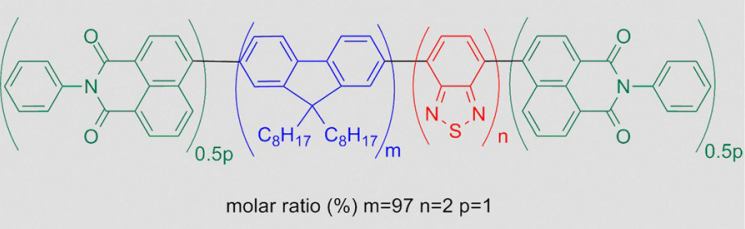

(b)

Fig. 11 Chemical structures of white electroluminescent copolymers with $\mathbf{a}$ blue and orange or $\mathbf{b}$ red, green and blue emitting units in the main chain

percentages of a red emitting osmium complex and green fluorescent benzothiadiazole units have been tethered to the main chain of a blue emitting polyfluorene (Fig. 12a). Red iridium phosphors have been also grafted as pendant groups to a similar polymeric skeleton made of blue fluorene type and green benzothiadiazole emitting units (Fig. 12b).

Hyperbranched white emitting copolymers composed of blue polyfluorene arms and orange star shape cores have also shown power efficiency higher than that observed for the linear counterparts since their branched structure prevents interchain interactions which induce emission quenching in linear polymers (Fig. 13) [34].

Less conventional systems have been also explored. For instance, a white electroluminescent network film has been recently used for the first time as the emissive layer in highly efficient and color-stable WOLEDs. In particular, during WOLED fabrication, three organic red, green and blue emitters mixed in electrolyte solution have been deposited onto the

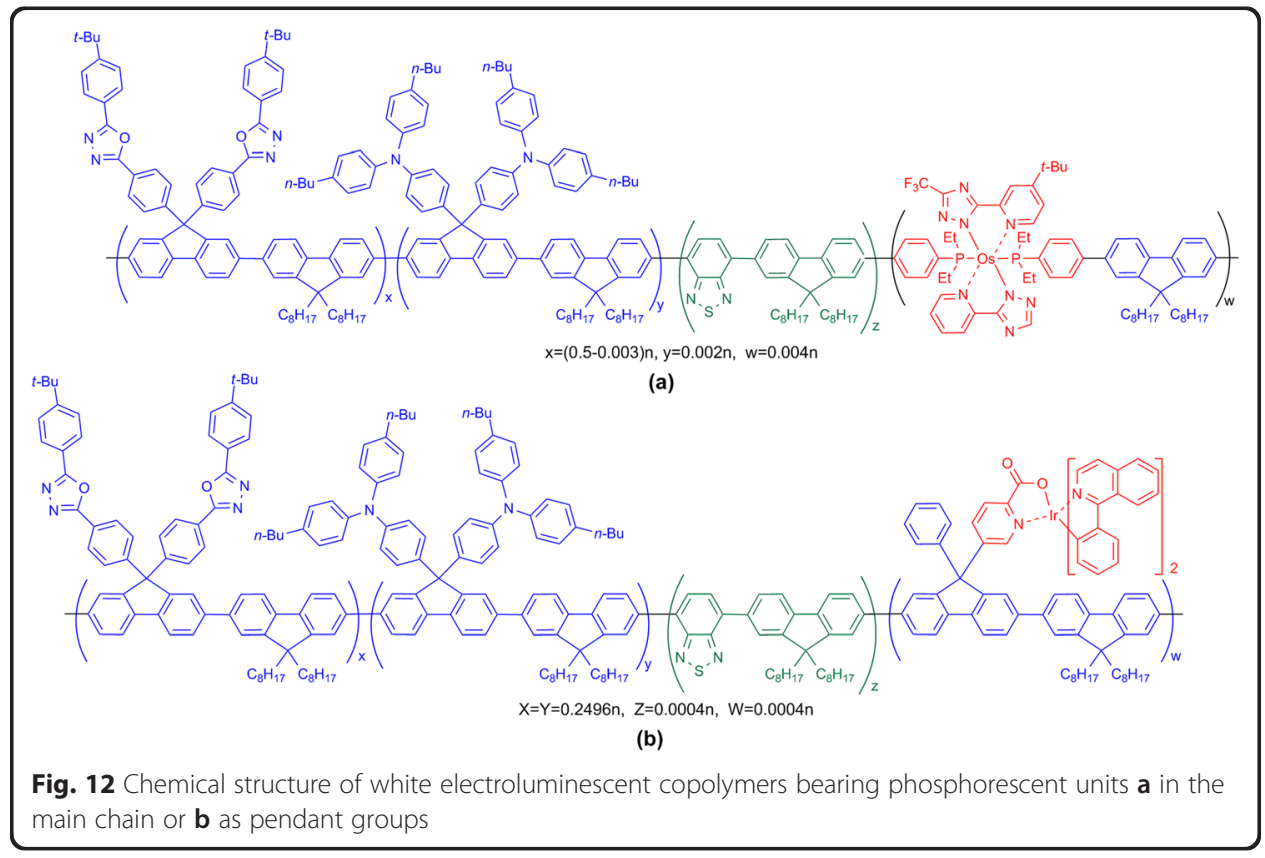




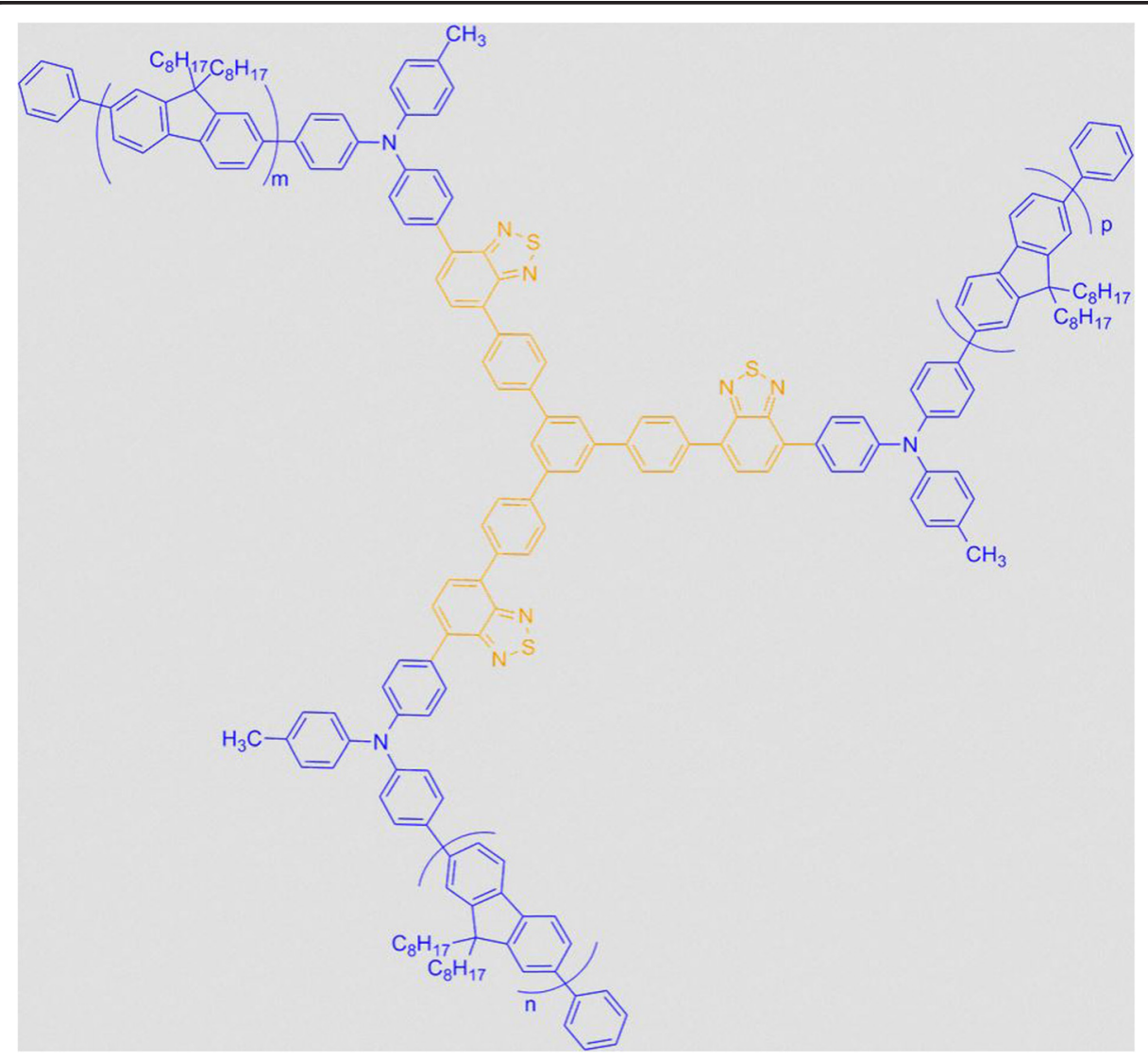

Fig. 13 Chemical structure of white electroluminescent star shape copolymer

device electrode and then oxidized by in situ electrochemical copolymerization strategy leading to a cross-linked polymer network film (Fig. 14) [35]. This film shows excellent structural and phase stability and no phase separation occurs between monomers since, due to their random oxidation, they are fixed and uniformly distributed in the cross-linked structure. In this way, WOLEDs with bright electroluminescence covering the whole visible range have been obtained.

\section{Approach (5): White light emission from inorganic LEDs and organic down converters}

To date, white inorganic LEDs have external quantum efficiencies higher than WOLEDs and are generally composed of a blue emitting inorganic LED coated by yellow or green-red inorganic color converters such as rare-earth element containing materials [36]. Despite their high stability and high color conversion efficiency, inorganic color converters suffer from high costs, non-uniform dispersion, difficult color tuning and relatively poor emission in the green and red spectral regions, resulting in typically low rendering of saturated colors.

An alternative approach to white electroluminescence, which is currently gaining particular attention by the scientific community, consists in coating a blue inorganic LED with a layer of one or more organic luminescent color converters (organic LUCO dyes). These hybrid device architectures combine the high efficiency of inorganic LEDs with the high color rendering index, good color tunability and potentially low manufacturing costs of organic converters. 


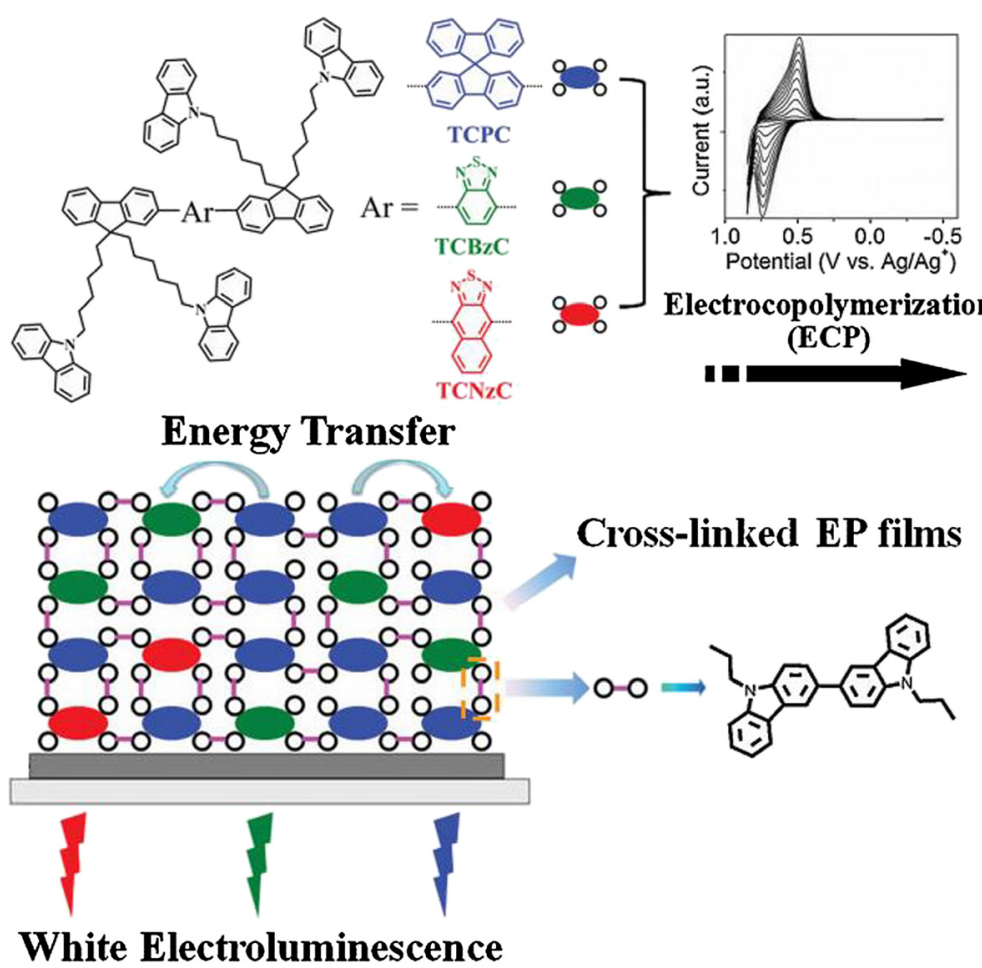

Fig. 14 Schematic representation of in situ electrochemical copolymerization technique. Adapted with permission from ref. [35]. Copyright 2014, WILEY-VCH Verlag GmbH \& Co. KGaA, Weinheim

In particular, the inorganic LED is used as both the blue emitting component and as the excitation source for organic LUCO dyes having broad emission spectra in the yellow or green-red visible region: the combined emission from the light source and the converter results in good quality white light output.

In order to tolerate the long lifetimes and high operational temperatures of the pump LEDs, organic color converters must have high temporal, thermal and photochemical stability. Moreover, to reduce their aggregation and emission quenching in the solid state, they are generally embedded into a non-emissive polymeric matrix.

For instance, white hybrid light emitting devices with CIE coordinates $(0.34,0.31)$ have been recently fabricated by depositing, on a blue inorganic LED, a solution of the yellow dye [BODFluTh $]_{2} \mathrm{FB}$ (Fig. 15) in 1,4-cyclohexanedimethanol divinyl ether (CHDV) containing a photoacid generator [37]. Under UV light exposure, CHDV is cured in a non emissive matrix which encapsulates the organic dye and generates a smooth solid dome on top of the LED chip. The resulting architecture has luminous efficiency four fold higher than the bare blue LED component, this showing that integration with the organic down converter influences both the final emission color and performances of the hybrid device.

In 2014, white light emitting hybrid devices with high color rendering index and efficiency over $90 \%$ have been also fabricated by a simple and fast immersion procedure of a commercial blue inorganic LED in a mold containing a partially pre-polymerized viscous mixture of methyl-methacrylate, the blue Coumarin 30 and the red DPPth organic down converters (Fig. 16) [38]. In this way, a dye doped polymethyl-methacrylate (PMMA) shell is generated by free radical polymerization reaction directly on top of 


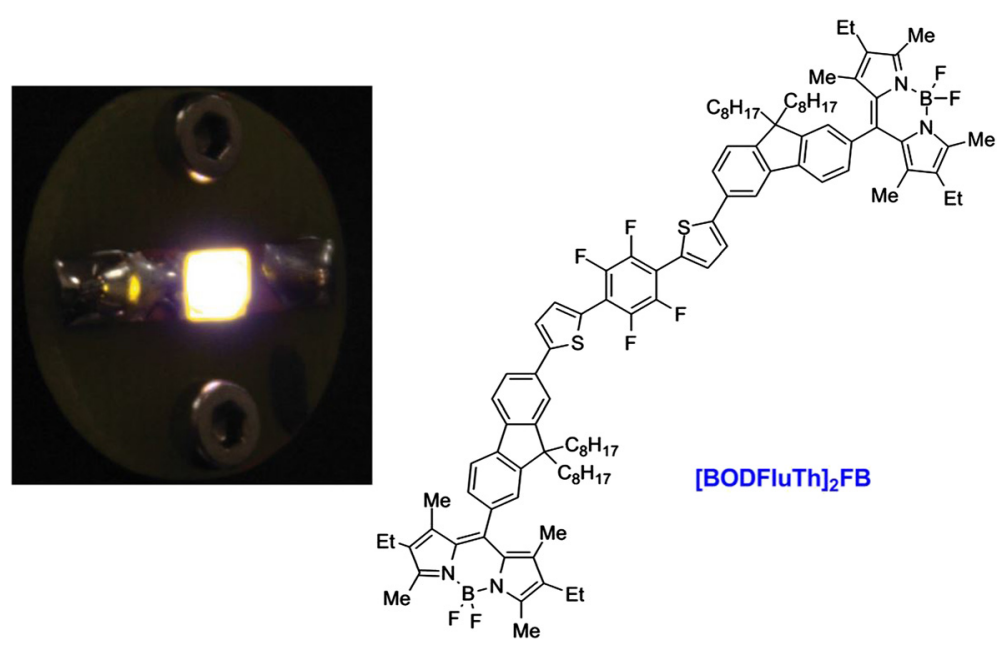

Fig. 15 Picture of the hybrid white LED based on the yellow organic LUCO dye [BODFluTh $]_{2} \mathrm{FB}$

the blue LED. This strategy is particularly appealing since it can be applied to commercial LEDs with light bulb design and can be easily scaled up to industrial lighting sources production.

The use of a polymer matrix which prevents the emission quenching of LUCO dyes is not always necessary: this is the case of organic down converters as the yellow dye BTPETD (Fig. 17) showing aggregate induced emission [39]. The aggregation of the dye molecules in thin films does not quench and, on the contrary, it enhances their photoluminescence in the solid state. Therefore, a layer of BTPETD can be directly deposited on top of a blue LED by drop casting from toluene solution. Hybrid white LEDs with CIE coordinates $(0.32,0.33)$ can be fabricated by carefully adjusting the thickness of this down conversion layer. Final encapsulation of the resulting hybrid device with an epoxy coating is anyway performed to improve their lifetime and stability.

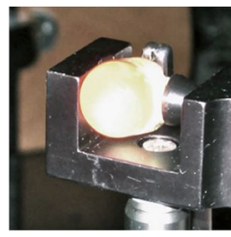

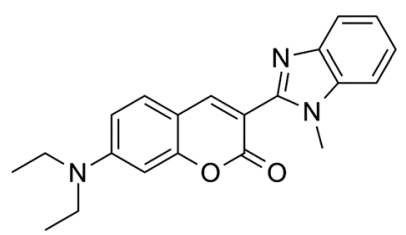

Coumarine 30

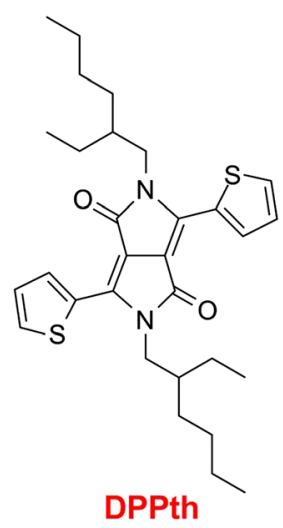

DPPth

Fig. 16 Picture of a hybrid white LED with light bulb design obtained by coating a commercial blue LED with a PMMA matrix doped with Coumarin 30 and DPPth LUCO dyes. White LED picture reproduced with permission from ref. [38]. Copyright 2014. Nature Publishing Group 


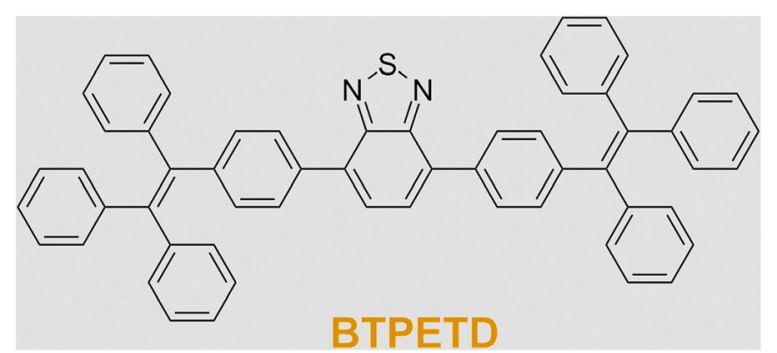

Fig. 17 Chemical structure of the yellow BTPETD dye showing aggregate induced emission

\section{Conclusions}

Considerable scientific efforts have been devoted so far to the research of organic and organometallic emitters for highly efficient white light emitting devices. WOLED technology may open a new concept of indoor/outdoor illumination architectures having the distinctive feature to be manufactured as flexible lighting sources with various shapes that emit good quality and uniform white light with low energy consumption.

Various approaches are available to achieve white electroluminescence from organic systems: among them, WOLEDs based on the combination of multiple emitters appear closer to practical applications with respect to those ones relying on more synthetically challenging single white emitting materials.

On another side, very recent studies have also demonstrated the possibility to combine highly efficient commercial inorganic LEDs with organic color converter dyes having high color rendering index. This new hybrid technology is readily expected to have deep impact on lighting market, due to lower fabrication costs with respect to fully inorganic white LEDs.

\section{Abbreviations}

ACL: Anode-cathode layer; CIE coordinates: Commission Internationale d'Eclairage chromaticity coordinates; ETL: Electron transporting layer; HBL: Hole blocking layer; HTL: Hole transporting layer; ITO: Indium tin oxide; LED: Light emitting diode; LEP: Light emitting polymer; LUCO: Luminescent color converter; PMMA: Polymethyl-methacrylate; PVK: Polyvinylcarbazole; RISC: Reverse intersystem crossing; SSL: Solid state lighting; TADF: Thermally activated delayed fluorescence; UV: Ultraviolet; WOLED: White organic light emitting diode.

\section{Competing interests}

The authors declare that they have no competing interests.

\section{Authors' contributions}

Both authors have contributed in the construction of the manuscript. Both authors read and approved the final manuscript.

\section{Authors' information}

G. M. Farinola is Professor of Organic Chemistry at the Chemistry Department of University of Bari. He received his PhD in Chemical Sciences in 1997 and, after holding a position as

Researcher, in 2002 he was appointed Associate Professor. In 2010 he got the qualification as a Full Professor. His research interests are mainly focused on synthesis and properties of $\pi$-conjugated materials for photonics and electronics. For his contributions in the development of synthetic methods for organic semiconductors in 2003 he was awarded the Ciamician Medal of the Italian Chemical Society for young organic chemists and in 2008 he received the CINMPIS Award for Innovation in Organic Synthesis.

R. Ragni graduated in Chemistry in 2001 and received her PhD in Chemical Sciences at the University of Bari (Italy) in 2004. In 2006, she was visiting researcher at the "Instituut voor Moleculaire Chemie- Universiteit van Amsterdam" (The Netherlands) and at the "Westfälische Wilhelms- Universität Münster Physikalisches Institut" (Germany). She presently holds a position as Researcher in Organic Chemistry at the University of Bari since 2008. Her research interests deal with the synthesis and properties of (electro) luminescent organic and organometallic compounds. 


\section{Acknowledgements}

The authors thank the Ministero dell'Istruzione dell'Università e della Ricerca (MIUR), the University of Bari "Aldo Moro" and the DHITECH-Distretto Tecnologico High-Tech S.c.a.r.I. (Project PONa3_00369 SISTEMA: "Laboratorio per lo Sviluppo Integrato delle Scienze e delle TEcnologie dei Materiali Avanzati e per dispositivi innovative" and Project PON02_00563_3316357 MAAT: "Molecular Nanotechnology for HeAlt and EnvironmenT").

Thematic series on the 14th International Symposium on the Science and Technology of Lighting LS14.

\section{Received: 8 February 2015 Accepted: 1 September 2015}

\section{Published online: 18 September 2015}

\section{References}

1. Reineke S, Lindner F, Schwartz G, Seidler N, Walzer K, Lussem B, et al. White organic light-emitting diodes with fluorescent tube efficiency. Nature. 2009;459:234-9.

2. Farinola GM, Ragni R. Electroluminescent materials for white organic light emitting diodes. Chem Soc Rev. 2011:40:3467-82

3. Taguchi T. Present status of energy saving technologies and future prospect in white LED lighting. IEEJ Trans. 2008;3:21-6.

4. Dawson TL. Development of efficient and durable sources of white light. Color Technol. 2010;126:1-10.

5. Kamtekar KT, Monkman AP, Bryce MR. Recent advances in white organic light-emitting materials and devices (WOLEDs). Adv Mater. 2010;22:572-82.

6. Beaupré $S$, Boudreault P-LT, Leclerc M. Solar-energy production and energy-efficient lighting: photovoltaic devices and white-light-emitting diodes using poly(2,7-fluorene), poly(2,7-carbazole), and poly(2,7-dibenzosilole) derivatives. Adv Mater. 2010;22:E6-27.

7. Raja IUH, Lee JY, Kim IT, Lee SH. Recent progress in the development of polymers for white light-emitting polymer devices. Monatsh Chem. 2008;139:725-37.

8. Takei Y. Energy saving lighting efficiency technologies. Sci Technol Trends-Quarterly Rev. 2009:32:59-71.

9. Ying $L, H o C-L, W u ~ H, C a O Y$, Wong $W$-Y. White polymer light-emitting devices for solid-state lighting: materials, devices, and recent progress. Adv Mater. 2014;26:2459-73.

10. Grimsdale AC, Leok Chan K, Martin RE, Jokisz PG, Holmes AB. Synthesis of light-emitting conjugated polymers for applications in electroluminescent devices. Chem Rev. 2009;109:897-1091.

11. Hung LS, Chen $\mathrm{CH}$. Recent progress of molecular organic electroluminescent materials and devices. Mater Sci Eng R. 2002;39:143-222

12. Evans RC, Douglas P, Winscom CJ. Coordination complexes exhibiting room-temperature phosphorescence: evaluation of their suitability as triplet emitters in organic light emitting diodes. Coord Chem Rev. 2006;250:2093-126.

13. Eliseevaa SV, Bünzli J-CG. Lanthanide luminescence for functional materials and bio-sciences. Chem Soc Rev. 2010;39:189-227.

14. Baldo MA, O'Brien DF, Thompson ME, Forrest SR. Excitonic singlet-triplet ratio in a semiconducting organic thin film. Phys Rev B Condens Matter. 1999;60:14422-8.

15. Kim S, Yook KS, Lee JY. Above $20 \%$ external quantum efficiency in novel hybrid white organic light-emitting diodes having green thermally activated delayed fluorescent emitter. Sci Reports. 2014;4(6019):1-6.

16. Tang KC, Tseng SR, Li WS, Meng HF, Horng SF, Hsu CS. Broad band and white phosphorescent polymer lightemitting diodes in multilayer structure. Synth Met. 2008;158:287-91.

17. Yook KS, Lee JY. Solution processed high efficiency blue and white phosphorescent organic light-emitting diodes using a high triplet energy exciton blocking layer. Org Electron. 2011;12:1293-7.

18. Sessolo M, Tordera D, Bolink HJ. Ionic iridium complex and conjugated polymer used to solution-process a bilayer white light-emitting diode. ACS Appl Mater Interfaces. 2013;5:630-4.

19. Wang Q, Ding J, Ma D, Cheng Y, Wang L, Wang F. Manipulating charges and excitons within a single-host system to accomplish efficiency/CRI/ color-stability trade-off for high-performance OWLEDs. Adv Mater. 2009;21:2397-401.

20. Nishide J, Nakanotani $\mathrm{H}$, Hiraga $Y$, Adachi C. High-efficiency white organic light-emitting diodes using thermally activated delayed fluorescence. Appl Phys Lett. 2014;104:233304-1-5.

21. Cho YJ, Yook KS, Lee JY. Cool and warm hybrid white organic light-emitting diode with blue delayed fluorescent emitter both as blue emitter and triplet host. Sci Reports. 2015;5(7859):1-7.

22. Sun JX, Zhu XL, Peng HJ, Wong M, Kwok HS. Bright and efficient white stacked organic light-emitting diodes. Org Electron. 2007:8:305-10.

23. Alam MM, Jenekhe SA. Binary blends of polymer semiconductors: nanocrystalline morphology retards energy transfer and facilitates efficient white electroluminescence. Macromol Rapid Commun. 2006;27:2053-9.

24. Yu L, Liu J, Hu SJ, He RF, Yang W, Wu HB, et al. Red, green, and blue light-emitting polyfluorenes containing a dibenzothiophene-S, S-dioxide unit and efficient high-color-rendering-index white-light-emitting diodes made therefrom. Adv Funct Mater. 2013;23:4366-76.

25. Kim T-H, Lee HK, Park OO, Chin BD, Lee S-H, Kim JK. White-light-emitting diodes based on iridium complexes via efficient energy transfer from a conjugated polymer. Adv Funct Mater. 2006;16:611-7.

26. Wu HB, Zhou GJ, Zou JH, Ho C-L, Wong W-Y, Yang W, et al. Efficient polymer white-light-emitting devices for solid-state lighting. Adv Mater. 2009;21:4181-4.

27. Mazzeo M, Vitale V, Della Sala F, Anni M, Barbarella G, Favaretto L, et al. Bright white organic light-emitting devices from a single active molecular material. Adv Mater. 2005;17:34-9.

28. Williams EL, Haavisto K, Li J, Jabbour GE. Excimer-based white phosphorescent organic light emitting diodes with nearly 100\%internal quantum efficiency. Adv Mater. 2007;19:197-202.

29. Liu D, Zhang Z, Zhang H, Wang Y. A novel approach towards white photoluminescence and electroluminescence by controlled protonation of a blue fluorophore. Chem Commun. 2013:49:10001-3.

30. Liu J, Zhou Q, Cheng Y, Geng Y, Wang L, Ma D, et al. White electroluminescence from a single-polymer system with simultaneous Two-color emission: polyfluorene as the blue host and a 2,1,3-benzothiadiazole derivative as the orange dopant. Adv Funct Mater. 2006;16:957-65. 
31. Lee P-I, Hsu SL-C, Lee R-F. White-light-emitting diodes from single polymer systems based on polyfluorene copolymers end-capped with a dye. Polymer. 2007:48:110-5.

32. Chien C-H, Liao S-F, Wu C-H, Shu C-F, Chang S-Y, Chi Y, et al. Electrophosphorescent polyfluorenes containing osmium complexes in the conjugated backbone. Adv Funct Mater. 2008;18:1430-9.

33. Wu F-I, Yang X-H, Neher D, Dodda R, Tseng Y-H, Shu C-F. Efficient white-electrophosphorescent devices based on a single polyfluorene copolymer. Adv Funct Mater. 2007;17:1085-92.

34. Chen $L$, Li PC, Tong $H$, Xie ZY, Wang $L X$, Jing XB, et al. White electroluminescent single-polymer achieved by incorporating three polyfluorene blue arms into a star-shaped orange core. J Polym Sci A Polym Chem. 2012;50:2854-62

35. Gu C, Fei T, Lv Y, Feng T, Xue SF, Lu D, et al. Color-stable white electroluminescence based on a cross-linked network film prepared by electrochemical copolymerization. Adv Mater. 2010;22:2702-5.

36. Nakamura S, Fasol G. The Blue Laser Diode: GaN Based Light Emitters and Lasers. Berlin: Springer; 1996. p. 216.

37. Findlay NJ, Bruckbauer J, Inigo AR, Breig B, Arumugam S, Wallis DJ, et al. An organic down-converting material for white-light emission from hybrid LEDs. Adv Mater. 2014;26:7290-4.

38. Di Martino D, Beverina L, Sassi M, Brovelli S, Tubino R, Meinardi F. Straightforward fabrication of stable white LEDs by embedding of inorganic UV-LEDs into bulk polymerized polymethyl-methacrylate doped with organic dyes. Sci Reports. 2014;4:4400/1-5.

39. Yue Z, Cheung YF, Choi HW, Zhao Z, Tang BZ, Wong KS. Hybrid GaN/Organic white light emitters with aggregation induced emission organic molecule. Opt Mater Express. 2013;3:1906-11.

\section{Submit your manuscript to a SpringerOpen ${ }^{\circ}$ journal and benefit from:}

- Convenient online submission

- Rigorous peer review

- Immediate publication on acceptance

- Open access: articles freely available online

- High visibility within the field

- Retaining the copyright to your article 